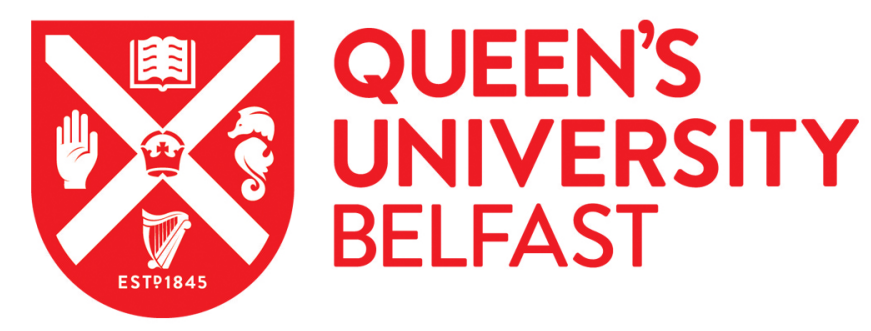

\title{
Comparison of Hydrodynamic Cavitation Devices Based on Linear and Swirling Flows: Degradation of Dichloroaniline in Water
}

\author{
Sarvothaman, V. P., Simpson, A., \& Ranade, V. V. (2020). Comparison of Hydrodynamic Cavitation Devices \\ Based on Linear and Swirling Flows: Degradation of Dichloroaniline in Water. Industrial and Engineering \\ Chemistry Research, 59(30), 13841-13847. https://doi.org/10.1021/acs.iecr.0c02125, \\ https://doi.org/10.1021/acs.iecr.0c02125
}

Published in:

Industrial and Engineering Chemistry Research

\section{Document Version:}

Peer reviewed version

Queen's University Belfast - Research Portal:

Link to publication record in Queen's University Belfast Research Portal

Publisher rights

Copyright 2020 ACS. This work is made available online in accordance with the publisher's policies. Please refer to any applicable terms of use of the publisher.

\section{General rights}

Copyright for the publications made accessible via the Queen's University Belfast Research Portal is retained by the author(s) and / or other copyright owners and it is a condition of accessing these publications that users recognise and abide by the legal requirements associated with these rights.

Take down policy

The Research Portal is Queen's institutional repository that provides access to Queen's research output. Every effort has been made to ensure that content in the Research Portal does not infringe any person's rights, or applicable UK laws. If you discover content in the Research Portal that you believe breaches copyright or violates any law, please contact openaccess@qub.ac.uk. 


\section{Comparison of Hydrodynamic Cavitation Devices based on Linear and Swirling Flows: Degradation of dichloroaniline in water}

Varaha Prasad Sarvothaman ${ }^{1}$, Alister Simpson ${ }^{2}$ and Vivek V. Ranade ${ }^{1,3^{*}}$

Multiphase Reactors \& Intensification Group (mRING)

${ }^{1}$ School of Chemistry and Chemical Engineering Queen's University Belfast, Belfast BT9 5AG, United Kingdom.

${ }^{2}$ School of Aerospace and Mechanical Engineering Queen's University Belfast, Belfast BT9 5AG, United Kingdom.

${ }^{3}$ Bernal Institute, University of Limerick, Limerick V94 T9PX, Ireland.

* Author to whom correspondence should be addressed Email: ‥Ranade@qub.ac.uk, Vivek.Ranade@ul.ie 


\begin{abstract}
Hydrodynamic cavitation $(\mathrm{HC})$ is being increasingly used for a wide range of applications including waste water treatment. No systematic comparison of pollutant degradation performance of different $\mathrm{HC}$ devices is available. In this work, for the first time: a basis for comparing performance of $\mathrm{HC}$ devices and a systematic comparison of pollutant degradation performance of five different types of $\mathrm{HC}$ devices based on linear and swirling flows is presented. 2,4 dichloroaniline (DCA) was selected as a model pollutant in water as it contains multiple functional groups on an aromatic ring. Experiments were performed at two values of pressure drop across HC devices (100 and $200 \mathrm{kPa}$ ) at a constant initial concentration (35 ppm), $\mathrm{pH}(7)$ and temperature $\left(18^{\circ} \mathrm{C}\right)$ for five types of $\mathrm{HC}$ devices namely orifice, venturi, orifice with swirl, venturi with swirl and vortex diode. The pollutant degradation was interpreted by a per-pass degradation factor approach. The study demonstrated that five different types of cavitation devices performed similar to each other when these devices were designed to exhibit similar pressure drop versus flow rate curve. It was conclusively shown that swirl does not supress degradation performance while offering advantages on shielding device walls from collapsing cavities. This is an important and new result which will be useful for selecting and designing cavitation devices. Pollutant degradation data for geometrically similar vortex diodes of two smaller scales showed significantly higher degradation performance. The number of passes required for $\sim 10 \%$ degradation for the devices with nominal capacity of 1, 5 and 20 LPM were 15, 100 and 1200 passes respectively. The presented experimental data from these seven devices will be useful for evaluating computational models and hopefully stimulate further development of predictive computational models in this challenging area.
\end{abstract}




\section{Introduction}

Hydrodynamic cavitation ( $\mathrm{HC}$ ) is a process of generation, growth and subsequent collapse of gas/vapour filled cavities. HC has been used in a variety of industrially relevant applications and has the potential to be scaled-up..$^{1-9}$ Several different types of $\mathrm{HC}$ devices have been used. ${ }^{1-9}$ In all of these $\mathrm{HC}$ devices, flow of liquids is arranged in such a way that in certain regions, local pressure approaches vapour pressure of liquid. Turbulent pressure fluctuations under such conditions generate cavitation. Such low pressure regions can be generated using either active devices (comprising moving parts like rotating or vibrating blades) or passive devices (without any moving parts like orifice or venturi). Active devices are expensive in terms of capital and operating costs. For applications like waste water treatment, where overall cost is one of the primary concerns, passive devices are often used. Two of the most commonly used conventional $\mathrm{HC}$ devices are orifice and venturi. It was shown recently that for these two $\mathrm{HC}$ devices, cavitation zone occurs adjacent to the solid walls. ${ }^{10}$ This causes erosion and $\mathrm{HC}$ device subsequently loses its effectiveness with time. For applications like waste water treatment, no maintenance and long life of equipment are often key requirements. Simpson and Ranade ${ }^{10}$ have recently shown that placing a swirler before orifice or venturi combines rotational flow and linear flow leading to pushing the cavitation zone away from solid walls. These designs look promising for extending the working life of HC devices. Unfortunately, no information is available on their performance in terms of pollutant degradation.

In fact, no systematic comparison of pollutant degradation performance of different HC devices is available. Some attempts on comparison of $\mathrm{HC}$ devices simply compare performance at constant pressure drop without discussing any basis for the same. In this work a basis for comparing performance of $\mathrm{HC}$ devices is discussed. Quantitative data on pollutant degradation performance of five different types of $\mathrm{HC}$ devices based on linear and swirling flows is presented. A complex organic pollutant comprising aromatic ring and multiple functions groups (2,4 dichloroaniline: DCA) was used as a model pollutant. A possibility of exploiting advantages of swirling flow such as shielding device walls from collapsing cavities without adverse influence on degradation performance is presented. The degradation performance of two smaller scales of vortex diode is also presented. The presented results provide an excellent starting point for establishing a consistent basis for comparing performance of different types of $\mathrm{HC}$ devices.

\section{Comparison of $\mathrm{HC}$ devices}

There are relatively few attempts where performance of different HC devices is compared by the same investigators using the same application. It will be worthwhile to examine different possible basis for 
comparing $\mathrm{HC}$ devices before briefly reviewing the previous work. Application of waste water treatment is considered in this work for comparing performance of different $\mathrm{HC}$ devices. The conclusions drawn from pollutant degradation will however provide general indication on the effectiveness of $\mathrm{HC}$ devices which may be valid for other applications as well.

Conventionally, batch experiments of pollutant degradation are interpreted using pseudo first order kinetics. ${ }^{8,11-12}$ This is rather misleading since the degradation is not a function of time but is a function of number of passes through HC device. Sarvothaman et al. ${ }^{13}$ have used per-pass degradation model to describe the batch experimental data of pollutant degradation. We believe that the per-pass degradation factor is the appropriate basis for comparing performance of $\mathrm{HC}$ devices. Once the perpass degradation factor is known for any $\mathrm{HC}$ device along with the operating pressure drop and flow rate, any other performance index of interest can be computed as:

Percentage degradation $=100 e^{-\varnothing n_{p}}$

Cavitational yield $(\mathrm{mg} / \mathrm{J})=\frac{\rho C_{0}}{\Delta P n_{p}}\left(1-e^{-\emptyset n_{p}}\right)$

Where $C_{0}\left(\mathrm{mg} / \mathrm{kg}\right.$ or ppm) is initial concentration of pollutant, $\phi$ is a per pass degradation factor, $n_{p}$ is number of passes through $\mathrm{HC}$ device and $\triangle \mathrm{P}(\mathrm{Pa})$ is pressure drop across $\mathrm{HC}$ device. Once the number of passes for the desired degradation and cavitational yield is known, cost of treatment can be calculated using the values of pump efficiency and price of electricity in a straight forward manner.

Per pass degradation factor for $\mathrm{HC}$ devices can be obtained by carrying out experiments. It is important to plan such experiments for evaluating relative performance of $\mathrm{HC}$ devices on a systematic basis. The five most obvious basis for carrying out such experiments are listed in Table 1.

Table 1: Possible basis for comparing per-pass performance of $\mathrm{HC}$ devices

\begin{tabular}{|c|c|c|c|}
\hline No. & Basis & Condition 1 & Condition 2 \\
\hline 1 & Hydrodynamic similarity & $R e_{1}=R e_{2}$ & $\frac{Q_{1}}{d_{t 1}}=\frac{Q_{2}}{d_{t 2}}$ \\
\hline 2 & Cavitation similarity & $C a_{1}=C a_{2}$ & $\frac{Q_{1}}{d_{t 1}^{2}}=\frac{Q_{2}}{d_{t 2}^{2}}$ \\
\hline 3 & $\begin{array}{c}\text { Same power consumption for } 1 \text { pass per } \\
1 \mathrm{~m}^{3} / \mathrm{s} \text { flow rate }\end{array}$ & $\Delta P_{1}=\Delta P_{2}$ & $E u_{1} \frac{Q_{1}^{2}}{d_{t 1}^{4}}=E u_{2} \frac{Q_{2}^{2}}{d_{t 2}^{4}}$ \\
\hline
\end{tabular}




\begin{tabular}{|c|c|c|c|}
\hline 4 & $\begin{array}{c}\text { Same power consumption per unit } \\
\text { volume of cavitation zone }\end{array}$ & $\frac{\Delta P_{1}}{d_{t 1}}=\frac{\Delta P_{2}}{d_{t 2}}$ & $E u_{1} \frac{Q_{1}^{2}}{d_{t 1}^{5}}=E u_{2} \frac{Q_{2}^{2}}{d_{t 2}^{5}}$ \\
\hline 5 & $\begin{array}{c}\text { Same power consumption per pass } \\
\text { through cavitation device }\end{array}$ & $\Delta P_{1} Q_{1}=\Delta P_{2} Q_{2}$ & $E u_{1} \frac{Q_{1}^{3}}{d_{t 1}^{4}}=E u_{2} \frac{Q_{2}^{3}}{d_{t 2}^{4}}$ \\
\hline
\end{tabular}

Where Euler number, Eu is defined as:

$$
E u=\frac{\Delta P}{0.5 \rho V_{t}^{2}}=\frac{\Delta P d_{t}^{4}}{0.5 \rho Q^{2}}
$$

Where $V_{t}$ and $d_{t}$ are characteristic velocity and length scales and $Q$ is flow rate through HC device $\left(=0.25 \pi d_{t}^{2} V_{t}\right)$. Unfortunately, not many published studies discuss the basis for comparison and do not report all the relevant quantities. In many cases, it is often difficult to unambiguously decide characteristic device dimension, $d_{t}$ (for example, a case of multiple slit orifice). The discharge coefficients or pressure drop versus flow characteristics are not adequately reported in many cases making the systematic comparison rather difficult. Key studies reporting comparison of $\mathrm{HC}$ devices are briefly discussed in the following.

Sivakumar and Pandit ${ }^{14}$ have compared performance (in terms of degradation of Rhodamine B dye in water) of six orifice plates at a same pressure drop across each device (30 psi or $\sim 207 \mathrm{kPa}$ ). Similar criteria of same pressure drop across HC devices was used for comparing performance of $i$ ) venturi and orifice ${ }^{11,15-18}$ and ii) vortex diode and orifice ${ }^{2}$ for different applications of cavitation. Mishra and Gogate $^{18}$ observed a nearly two-fold increase in performance for venturi over the orifice, when degradation was quantified on the basis of number of passes through cavitation device at same pressure drop. Carpenter et al..$^{19}$ used two pairs of orifice's and a pair of venturi's with comparable flowrates for studying emulsification of mustard oil in water. They observed a similar performance in terms of droplet size reduction with respect to number of passes for all the HC devices. A study comparing an orifice and vortex diode for the desulphurisation application has been reported. ${ }^{2}$ The study reported higher cavitation performance of vortex diode compared to orifice in terms of cavitation yield. In a recent study by Doltade et al. ${ }^{20}$ compared two venturi's for degradation of an industrial effluent over the range of $300-700 \mathrm{kPa}$. They report a superior performance for one of the venturi's by a factor of 4 at 300 and $500 \mathrm{kPa}$. At $700 \mathrm{kPa}$ the performances were reported to be similar. It must be noted that the initial concentration was different for the experiments carried out at different pressure drop conditions. Influence of initial concentration on degradation performance was not reported; making the direct comparison rather ambiguous. 
Recently, Simpson and Ranade ${ }^{10}$ have reported detailed hydrodynamic characteristics of five different HC devices: two of the most commonly utilized cavitation devices namely orifice and venturi which are based on linear flow, a high swirl flow device (vortex diode) and modified orifice and venturi with a swirler which use combination of swirling and linear flows for realising cavitation. These five devices were specially designed to ensure that they exhibit almost the same pressure drop versus flow rate curve. This unique set of devices therefore offer an interesting platform to compare their performance. In this work, we compare performance of these five devices in terms of pollutant degradation in water.

2,4 dichloroaniline (DCA) was used as a model pollutant. The DCA has two functional groups (chlorine and amine) and an aromatic ring. Chloro-organic compounds form an important class of pollutants. ${ }^{21}$, ${ }^{22}$ Many industrial effluent streams contain phenol/aniline, as these two serve as a precursor to many pharmaceutical/chemical manufacturing processes. The derivative compounds of dichloroaniline find its use in the production of dyes and herbicides. ${ }^{23}$ The DCA belong to a class of persistent environmental pollutants. ${ }^{24}$ The DCA degradation results presented here will complement the hydrodynamic results of Simpson and Ranade. ${ }^{10}$

\section{Experimental}

Simpson and Ranade ${ }^{10}$ have discussed role of swirling flows in altering characteristics of cavitation (by pushing the cavitation region away from the walls). The five types of hydrodynamic cavitation devices investigated by Simpson and Ranade ${ }^{10}$ were used in this study to carry out experiments on degradation of DCA in water. The devices were named as D1 to D5 as described in Table 2. Geometric and other details of these HC devices may be found in Simpson and Ranade ${ }^{10}$ and from Figure S.1 of the Supplementary Information. Two additional devices - smaller vortex based cavitation devices (vortex diodes with throat diameters, $d_{t}$ as $6 \mathrm{~mm}$ and $3 \mathrm{~mm}$ ) were also studied (denoted by D6 and D7 in Table 2). The pressure drop versus flow data for these devices is shown in Figure 1. Other than these seven devices, the data for devices from previous studies ${ }^{11,20}$ is also included in Figure 1.

The experimental set-up reported in Sarvothaman ${ }^{25}$ was used to operate these devices for carrying out the pollutant degradation experiments. The operation of these devices was reported without a by-pass line and degradation experiments were carried out at room temperature $\left(T=18 \pm 2{ }^{\circ} \mathrm{C}\right)$. In typical experiments, tap water with a calculated quantity of DCA stock solution was recirculated through the cavitation setup to ensure proper mixing. The concentration of DCA was analysed using UV-Vis spectroscopy. Typical spectroscopy profiles and the corresponding calibration curve are shown 
in Figure S.2. It was found that the characteristic peak at $241 \mathrm{~nm}$ gets saturated above $0.035 \mathrm{~g} / \mathrm{L}$ (35 $\mathrm{ppm})$. The initial concentration was therefore set to nearly $0.035 \mathrm{~g} / \mathrm{L}$ for all the experiments, to avoid dilution of the samples during analysis. The initial $\mathrm{pH}(\sim 7)$ of the DCA solution was left unaltered during the entire duration of experiments. Experiments were carried out at two values of pressure drops: $100 \mathrm{kPa}$ and $200 \mathrm{kPa}$ for all the seven devices. It was ensured that cavitation inception in all the devices occur at less than $100 \mathrm{kPa}$ pressure drop, this was ensured by manually tracking cavitation noise with the help of a stethoscope (Acoustica Deluxe Lightweight Dual Head Stethoscope, supplied by MDF instruments). Similar to most of the studies in literature, batch experiments were carried out to study degradation of DCA in water by circulating the water through HC devices. All experiments were carried out in triplicates. Samples were taken at dedicated time intervals. The average and standard error for concentration profile was calculated from the degradation data from replicate experiments. The confidence intervals for $\phi_{\mathrm{o}}$ were calculated using Minitab 19.

Table 2: Key characteristics of HC devices studied in this work, Orifice: $d_{t}=4 \mathrm{~mm}$; Venturi: $d_{t}=5 \mathrm{~mm}$; Degradation rate calculations are based on $C_{0}=1000 \mathrm{ppm}$ and $\rho=1000 \mathrm{~kg} / \mathrm{m}^{3}$

\begin{tabular}{|c|c|c|c|c|c|}
\hline $\begin{array}{c}\text { Device } \\
\text { name }\end{array}$ & $\begin{array}{c}\text { Device } \\
\text { description }\end{array}$ & $\begin{array}{c}\text { Inception } \\
\Delta \mathrm{P}(\mathrm{kPa})\end{array}$ & $\begin{array}{c}\Delta \mathrm{P}=\mathrm{aQ}^{2} ; \text { Value } \\
\text { of a }\left(\mathrm{kPa} / \mathrm{LPM}^{2}\right)\end{array}$ & $\begin{array}{c}\text { Initial degradation } \\
\text { Factor, } \phi_{0} \times 10^{5}\end{array}$ & $\begin{array}{c}\text { Initial Cavitational } \\
\text { yield @ 200 kPa, } \\
\mathrm{mg} / \mathrm{J}\end{array}$ \\
\hline D1 & Orifice & $80-90$ & 0.6 & $7.9 \pm 1.0$ & 37.5 \\
\hline D2 & Swirler + Orifice & $70-80$ & 0.6 & $8.4 \pm 1.8$ & 42.6 \\
\hline D3 & Venturi & $50-60$ & 0.6 & $9.9 \pm 1.4$ & 52.2 \\
\hline D4 & Swirler + Venturi & $50-60$ & 0.6 & $9.6 \pm 2.4$ & 48.6 \\
\hline D5 & $\begin{array}{c}\text { Vortex diode } \\
\mathrm{d}_{\mathrm{t}}=12 \mathrm{~mm}\end{array}$ & $50-80$ & 0.6 & $8.6 \pm 0.7$ & 51.6 \\
\hline D6 & $\begin{array}{c}\text { Vortex diode } \\
\mathrm{d}_{\mathrm{t}}=6 \mathrm{~mm}\end{array}$ & $50-80$ & 10 & $129.5 \pm 9.5$ & 540.5 \\
\hline D7 & $\begin{array}{c}\text { Vortex diode } \\
\mathrm{d}_{\mathrm{t}}=3 \mathrm{~mm}\end{array}$ & $80-100$ & 150 & $692.6 \pm 62.8$ & 3518.5 \\
\hline
\end{tabular}

\section{Results and discussion}

The experimental results of DCA degradation were processed using per pass degradation model. It was observed that the per pass degradation model of Sarvothaman et al. ${ }^{13}$ which was based on the assumption of constant per pass degradation factor over the number of passes may not be adequate for describing degradation of complex pollutants like DCA. Degradation of complex organic pollutants like DCA progresses through formation of several intermediates which may have different reactivity compared to the original pollutant. Hence in such cases there is a need to relax the assumption of a constant per-pass degradation factor to account for changing reactivity of intermediates. Recently 
Sarvothaman ${ }^{25}$ extended the per-pass degradation model to account for a possibility of varying per pass degradation factor. The extended per-pass model of Sarvothaman ${ }^{25}$ was used in this work to interpret the performance of $\mathrm{HC}$ devices. Considering that the number of passes, $\mathrm{n}_{\mathrm{p}}=\frac{Q}{V} t$, the overall behaviour of a typical cavitation-based water treatment experiments can be modelled as:

$$
Q \frac{d C}{d n_{p}}=-Q C+Q(1-\varnothing) C
$$

Where $\mathrm{C}$ is a concentration of pollutant, $\mathrm{V}$ is a holding tank volume, $\mathrm{Q}$ is a flow rate through cavitation device and $\phi$ is a per-pass degradation factor.

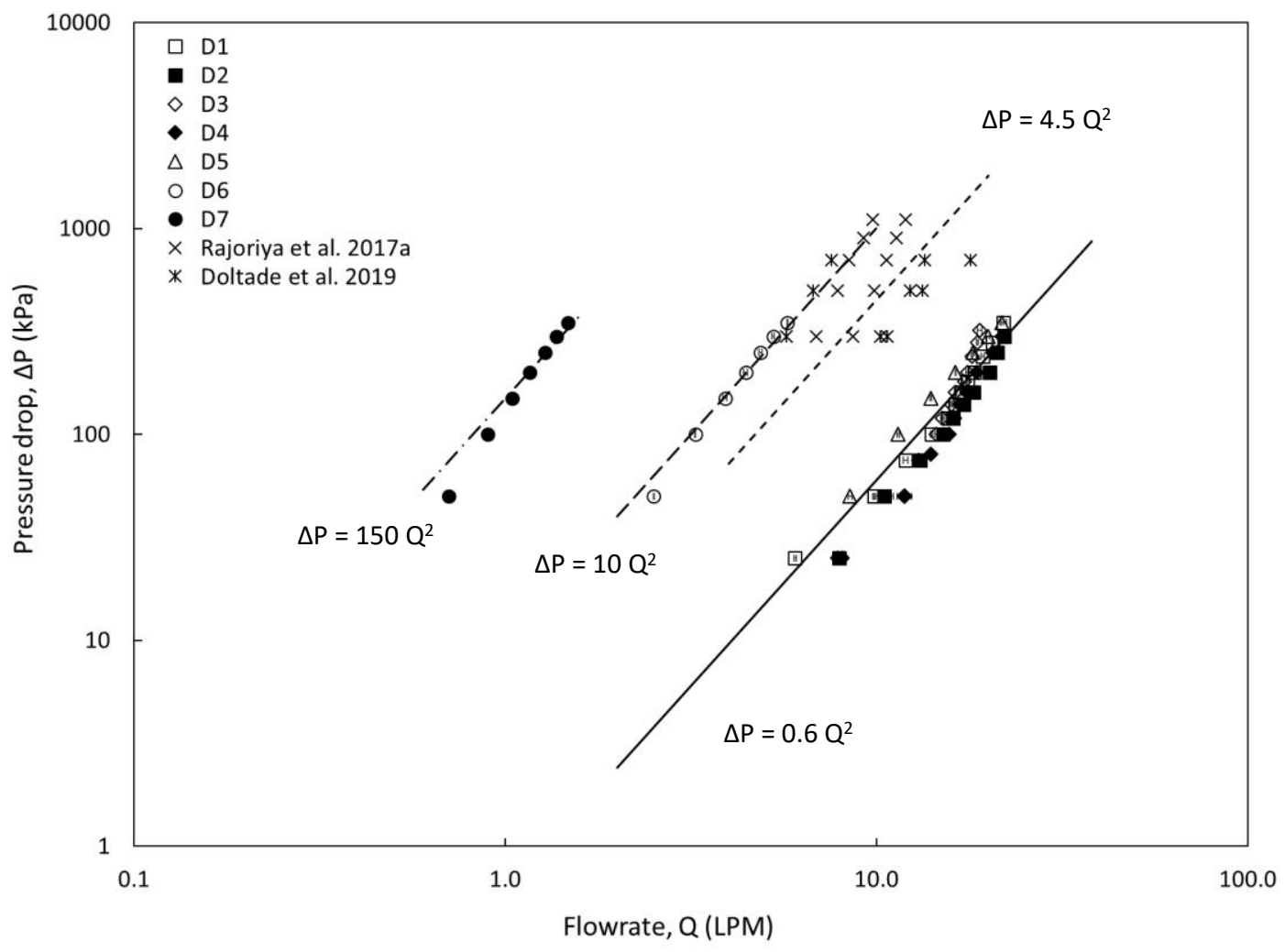

Figure 1: Pressure drop and flow relationship for hydrodynamic cavitation devices (Devices from current study: D1 to D7; devices from literature: Rajoriya et al. ${ }^{11}$, Doltade et al. ${ }^{20}$ ).

The per-pass degradation factor will depend on the generation rate of hydroxyl radicals (G), flow rate through cavitation device (Q), chemical nature of pollutant (reactivity with hydroxyl radicals, $\mathrm{k}_{2}$ ), intermediates formed during the radical reactions, concentration of the pollutant (C), concentration of scavengers $\left(C_{s}\right)$ and the relative rate reactivity of pollutant and scavengers $\left(k_{2} / k_{s}\right)$ with hydroxyl 
radicals. Following the model of Sarvothaman ${ }^{25}$, the following equation was used to fit the observed DCA concentration data:

$$
\left(\frac{C}{C_{0}}\right)=e^{-\emptyset_{0} n_{p} /\left(1+\emptyset_{0} n_{p}\right)}
$$

Further details of the model may be obtained from our previous work. ${ }^{25}$ The experimentally observed degradation profile of DCA obtained with device D1 operated at 100 and $200 \mathrm{kPa}$ is shown in Figure S.3. It can be seen that the data obtained at two pressure drops falls within error bars and influence of pressure drop across the device is not distinguishable. The experimental data obtained at two different pressure drops was therefore processed together for five types of devices exhibiting similar pressure drop versus flow rate behaviour (devices D1 to D5). The experimental data is shown in Figure 2 which confirm that influence of pressure drop is not distinguishable for any of these devices. The values of initial per pass degradation factors, $\phi_{\mathrm{o}}$ obtained for these five devices are reported in Table 2. It can be seen that degradation performance observed for all these five devices is comparable despite significantly different design of each device. A single value of $\phi_{\mathrm{o}}$ (average of $\phi_{\mathrm{o}}$ values reported in Table 2) was able to describe the experimental data reasonably well (see Figure 2). It should be noted that presence of swirler before orifice and venturi has not adversely affected degradation performance. Swirling flows offers benefits of realising cavitation zone away from device walls and therefore prolonging working life of $\mathrm{HC}$ devices. The device types (orifice, venturi and vortex diode) also apparently has no influence on observed degradation performance. This is indeed an interesting result and will offer an excellent validation case for computational models aiming to simulate $\mathrm{HC}$ devices and processes.

Considering that the per-pass degradation factor is changing with number of passes, it may be easier to simply compare initial cavitational yield rather than using the Equation (2). The initial cavitation yield can be calculated as:

Initial cavitational yield $(\mathrm{mg} / \mathrm{J})=\frac{\rho C_{0} \emptyset_{0}}{\Delta P}$

It can thus be seen that if the per pass device performance $\left(\phi_{0}\right)$ is not affected by pressure drop, the initial cavitation yield at $100 \mathrm{kPa}$ will be double of that at $200 \mathrm{kPa}$. The calculated values of initial cavitational yield at $200 \mathrm{kPa}$ are reported in Table 2 (by considering $C_{0}$ as $1000 \mathrm{ppm}$ ). For waste water treatment, the initial cavitation yield offers a useful measure of $\mathrm{HC}$ device performance and can be used for comparing different devices in addition to just per pass degradation factor $\left(\phi_{\mathrm{o}}\right)$. 


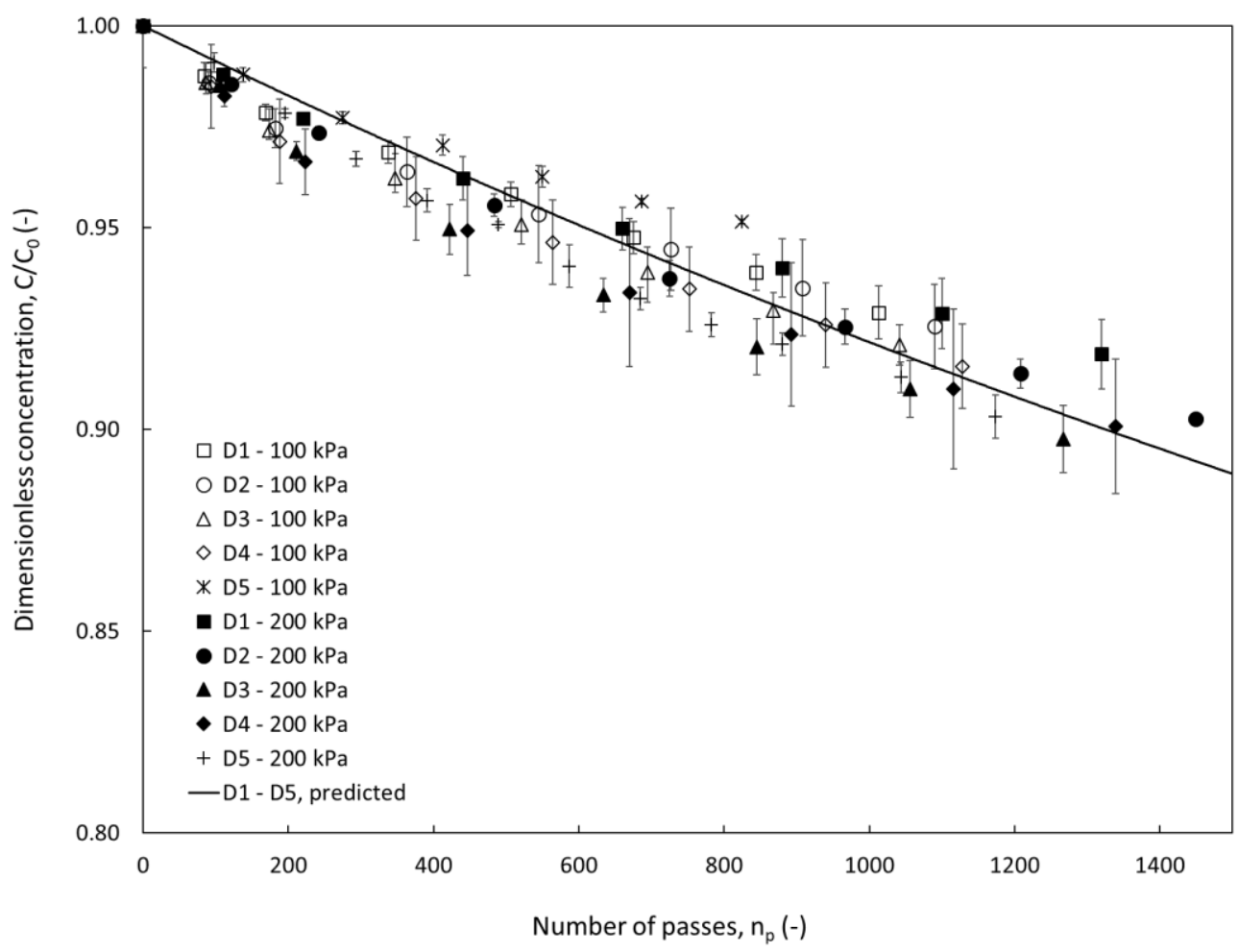

Figure 2: Influence of device type on degradation at 100 and $200 \mathrm{kPa}$ for devices D1 - D5 (Symbols: experimental data; Solid curves: predicted with Equation (5) $-\phi_{\circ}=8.89 \times 10^{-5}$ ).

An additional set of experiments were performed with two geometrically similar vortex diodes, with smaller characteristic dimension ( $d_{t}=6$ and $3 \mathrm{~mm}$ - devices D6 and D7 respectively). The experiments were performed at identical pressure drop conditions: 100 and $200 \mathrm{kPa}$. These two devices have same Euler number as that of Device 5. However, because of the smaller size of these devices, the pressure drop versus flow rate relationship is significantly different (see Figure 1 and Table 2). The observed DCA degradation data for devices D6 and D7 is compared with the average of devices D1 to D5 in Figure 3. It can be seen that these smaller devices with significantly lower flow rates exhibit significantly better degradation performance. Thus, it can be seen that having geometric similarity and same Euler number does not lead to similar degradation performance. A decrease in degradation performance with increase in device scale has been previously reported (Sarvothaman et al. ${ }^{13}$; Sarvothaman et $\left.a .{ }^{26}\right)$ and the present data is consistent with earlier studies.

Unfortunately, it was not possible to scale down swirler + orifice/ venturi which will mimic pressure drop versus flow rate curves of smaller vortex diodes. The data is not yet adequate to unambiguously conclude that $\mathrm{HC}$ devices having same pressure drop versus flow rate curves will lead to similar performance. It however offers a useful basis for further work. The data can be used to evaluate 
detailed computational fluid dynamics (CFD) based models to simulate devices (for example, along the lines of the model presented by Sarvothaman et $a{ }^{26}{ }^{26}$ ). The study by Simpson and Ranade ${ }^{10}$ and Sarvothaman et al. ${ }^{26}$ may provide an initial starting point for understanding trends of turbulence parameters/cavity trajectories in these five devices and different scales of vortex diode. The interpretation of obtained degradation results with help of such multi-scale models will be useful to develop better understanding of observed differences and similarities in performance of different $\mathrm{HC}$ devices. It is however, beyond the scope of this short note. The data of flow and per-pass degradation can be used for designing a continuous water treatment process based on hydrodynamic cavitation treatment following the procedure discussed by Sarvothaman et $a l^{13}$

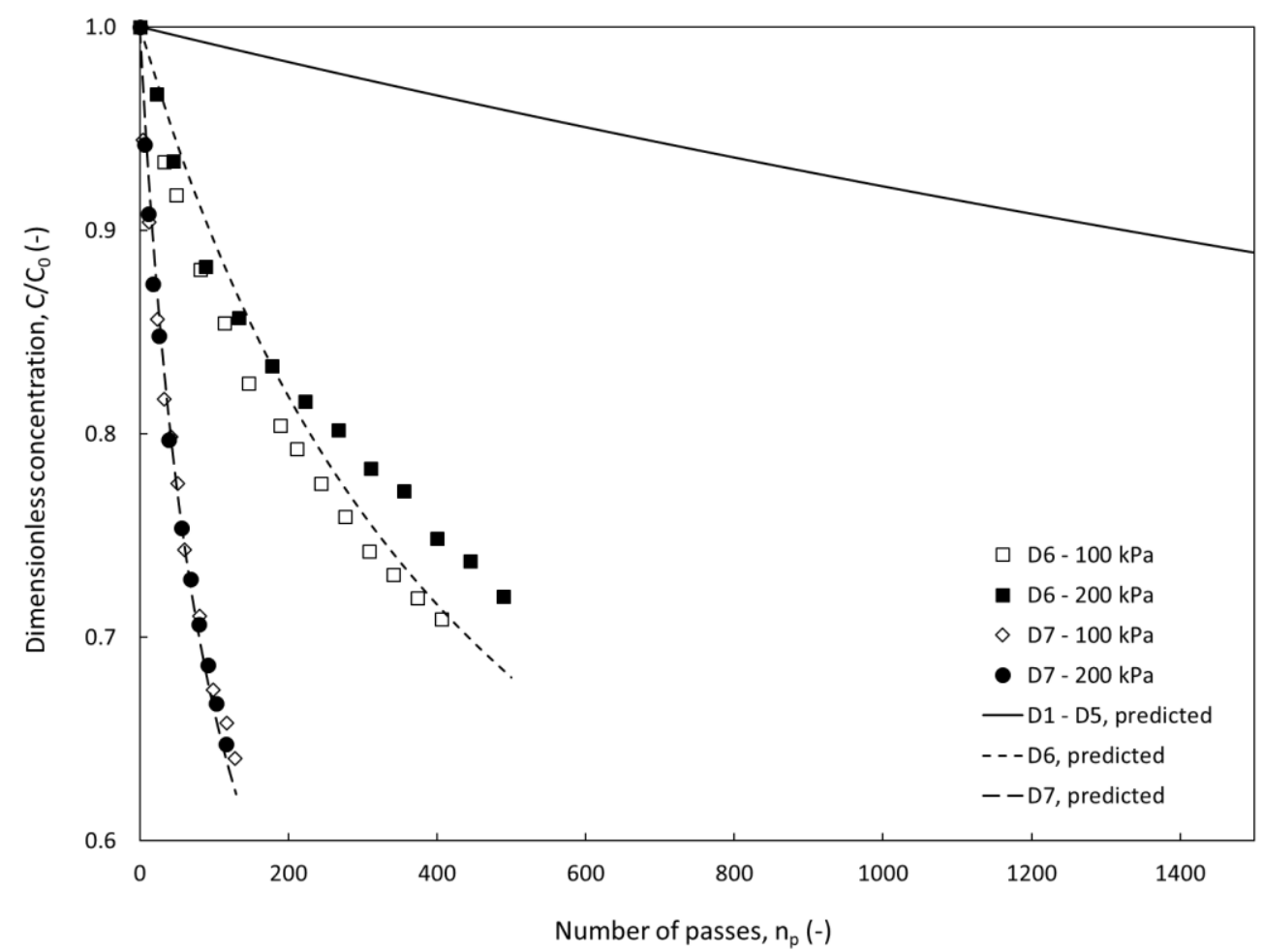

Figure 3: Influence of pressure drop on degradation for devices D6 and D7 (Symbols: experimental data; Solid curves: predicted with Equation (5) - $\phi_{\mathrm{o}}$ for D1 - D5 $=8.89 \times 10^{-5}, \phi_{\mathrm{o}}$ for D6 $=1.23 \times 10^{-3}$ $\phi_{\circ}$ for D7 $=6.92 \times 10^{-3}$ ).

\section{Summary and conclusions}

Degradation of DCA in water was investigated using seven $\mathrm{HC}$ devices in order to understand the basis for comparison of such devices. Five different types of HC devices (orifice, venturi, swirler + orifice, swirler + venturi and vortex diode) exhibiting almost the same pressure drop versus flow rate curves were considered. The degradation was interpreted with the help of a variable per-pass degradation factor model developed by Sarvothaman. ${ }^{25}$ Additional experiments were performed with two smaller 
vortex based $\mathrm{HC}$ devices having substantially different pressure drop versus flow rate curves. The key conclusions from the study are as follows:

- The degradation profile was not influenced by operating pressure drop across HC devices (100 $\mathrm{kPa}$ or $200 \mathrm{kPa}$ ) for any of the seven $\mathrm{HC}$ devices used in this study

- The degradation for the five types of HC devices (D1 - D5), i.e. with similar pressure drop versus flow rate curves could be described by a single value of per-pass degradation factor

- The introduction of swirl does not supress the degradation performance for orifice and venturi cavitation devices

- The degradation profiles of devices D6 and D7, with different pressure drop versus flow rate curves were drastically different than those for larger devices (D1 - D5)

Various possibilities of planning experiments for comparing performance of different $\mathrm{HC}$ devices are listed in Table 1. The presented results for the comparison of HC devices based on linear flows (orifice and venturi) and swirling flows (vortex diode) on a systematic basis complements the previous study on hydrodynamics for these devices (Simpson and Ranade ${ }^{10}$ ). The presented results will be useful for modellers as well as scientists and engineers interested in developing better HC devices.

\section{Supporting information}

Figures are presented for i) schematic of cavitation devices used in this study, ii) the UV-Vis spectroscopy calibration curve for dichloroaniline and iii) influence of pressure drop on degradation for the orifice device.

\section{Conflict of Interest Disclosure}

The authors declare the following competing financial interest(s): One of the authors (V.V.R.) is a founder/director of Vivira Process Technologies Pvt. Ltd., which commercially offers cavitation devices used in this study.

\section{Acknowledgements}

The authors gratefully acknowledge funding support from the start-up Grant G1013CHM from Queen's University Belfast, United Kingdom. VS would like to thank Mr. Kirin Hill for the assistance in wiring of three phase pump. 


\section{Notations}

Ca Cavitation number

C Concentration of pollutants in experiments (ppm)

$\mathrm{C}_{0} \quad$ Starting concentration of pollutants in experiments (ppm)

$\mathrm{C}_{\mathrm{s}} \quad$ Concentration of scavengers $\left(\mathrm{kmol} / \mathrm{m}^{3}\right)$

D1 Orifice based cavitation device

D2 Orifice-with-swirl based cavitation device

D3 Venturi based cavitation device

D4 Venturi-with-swirl based cavitation device

D5 Vortex-based cavitation device with $d_{t}=12-\mathrm{mm}$

D6 Vortex-based cavitation device with $d_{t}=6-\mathrm{mm}$

D7 Vortex-based cavitation device with $d_{t}=3-\mathrm{mm}$

Eu Euler number

$k_{2} \quad$ Second order rate constant $\left(\mathrm{M}^{-1} \mathrm{~s}^{-1}\right)$

$\mathrm{k}_{\mathrm{s}} \quad$ Rate constant for scavengers $\left(\mathrm{M}^{-1} \mathrm{~s}^{-1}\right)$

$\mathrm{n}_{\mathrm{p}} \quad$ Number of passes through cavitation device (-)

Q Recirculating flow through cavitation device $\left(\mathrm{m}^{3} / \mathrm{s}\right)$

Re Reynolds number

t Operation time (s)

$\mathrm{T} \quad$ Operating temperature $\left({ }^{\circ} \mathrm{C}\right)$

$\mathrm{V} \quad$ Volume of holding tank (L)

$\Delta \mathrm{P} \quad$ Pressure drop across cavitation device $(\mathrm{kPa})$

\section{Greek Symbols}

$\phi \quad$ Per-pass performance (or degradation) factor (-)

$\phi_{0} \quad$ Initial per-pass performance (or degradation) factor (-) 


\section{References}

1. Burzio, E., Bersani, F., Caridi, G.C.A., Vesipa, R., Ridolfi, L. and Manes, C., 2019. Water disinfection by orifice-induced hydrodynamic cavitation. Ultrasonics sonochemistry, 104740.

2. Suryawanshi, N.B., Bhandari, V.M., Sorokhaibam, L.G. and Ranade, V.V., 2017. Developing technoeconomically sustainable methodologies for deep desulfurization using hydrodynamic cavitation. Fuel, 210, 482-490.

3. Kumar, M.S., Sonawane, S.H., Bhanvase, B.A. and Bethi, B., 2018. Treatment of ternary dye wastewater by hydrodynamic cavitation combined with other advanced oxidation processes (AOP's). Journal of Water Process Engineering, 23, 250-256.

4. Nakashima, K., Ebi, Y., Shibasaki-Kitakawa, N., Soyama, H. and Yonemoto, T., 2016. Hydrodynamic cavitation reactor for efficient pretreatment of lignocellulosic biomass. Industrial \& Engineering Chemistry Research, 55(7), 1866-1871.

5. Baradaran, S. and Sadeghi, M.T., 2020. Desulfurization of Non-Hydrotreated Kerosene using Hydrodynamic Cavitation Assisted Oxidative Desulfurization (HCAOD) Process. Journal of Environmental Chemical Engineering, 103832.

6. Holkar, C.R., Jadhav, A.J., Pinjari, D.V. and Pandit, A.B., 2019. Cavitationally driven transformations: A technique of process intensification. Industrial \& Engineering Chemistry Research, 58(15), 5797-5819.

7. Dular, M., Griessler-Bulc, T., Gutierrez-Aguirre, I., Heath, E., Kosjek, T., Klemenčič, A.K., Oder, M., Petkovšek, M., Rački, N., Ravnikar, M. and Šarc, A., 2016. Use of hydrodynamic cavitation in (waste) water treatment. Ultrasonics sonochemistry, 29, 577-588.

8. Ranade, V. V.; Bhandari, V. M. Industrial wastewater treatment, recycling and reuse; ButterworthHeinemann: Oxford, England, 2014.

9. Gogate, P.R. and Pandit, A.B., 2004. Sonochemical reactors: scale up aspects. Ultrasonics Sonochemistry, 11(3-4), 105-117.

10. Simpson, A. and Ranade, V.V., 2019. 110th Anniversary: Comparison of Cavitation Devices Based on Linear and Swirling Flows: Hydrodynamic Characteristics. Industrial \& Engineering Chemistry Research, 58(31), 14488-14509.

11. Rajoriya, S., Bargole, S. and Saharan, V.K., 2017. Degradation of a cationic dye (Rhodamine 6G) using hydrodynamic cavitation coupled with other oxidative agents: Reaction mechanism and pathway. Ultrasonics sonochemistry, 34, 183-194.

12. Capocelli, M., Musmarra, D., Prisciandaro, M. and Lancia, A., 2014. Chemical effect of hydrodynamic cavitation: simulation and experimental comparison. AIChE Journal, 60(7), 25662572.

13. Sarvothaman, V.P., Nagarajan, S. and Ranade, V.V., 2018. Treatment of Solvent-Contaminated Water Using Vortex-Based Cavitation: Influence of Operating Pressure Drop, Temperature, Aeration, and Reactor Scale. Industrial \& Engineering Chemistry Research, 57(28), 9292-9304.

14. Sivakumar, M. and Pandit, A.B., 2002. Wastewater treatment: a novel energy efficient hydrodynamic cavitational technique. Ultrasonics sonochemistry, 9(3), 123-131.

15. Bargole, S., George, S. and Saharan, V.K., 2019. Improved rate of transesterification reaction in biodiesel synthesis using hydrodynamic cavitating devices of high throat perimeter to flow area ratios. Chemical Engineering and Processing-Process Intensification, 139, 1-13.

16. Rajoriya, S., Bargole, S. and Saharan, V.K., 2017. Degradation of reactive blue 13 using hydrodynamic cavitation: Effect of geometrical parameters and different oxidizing additives. Ultrasonics sonochemistry, 37, 192-202.

17. Prajapat, A.L. and Gogate, P.R., 2015. Intensification of depolymerization of aqueous guar gum using hydrodynamic cavitation. Chemical Engineering and Processing: Process Intensification, 93, 1-9.

18. Mishra, K.P. and Gogate, P.R., 2010. Intensification of degradation of Rhodamine B using hydrodynamic cavitation in the presence of additives. Separation and Purification Technology, 75(3), 385-391. 
19. Carpenter, J., George, S. and Saharan, V.K., 2017. Low pressure hydrodynamic cavitating device for producing highly stable oil in water emulsion: Effect of geometry and cavitation number. Chemical Engineering and Processing: Process Intensification, 116, 97-104.

20. Doltade, S.B., Dastane, G.G., Jadhav, N.L., Pandit, A.B., Pinjari, D.V., Somkuwar, N. and Paswan, R., 2019. Hydrodynamic cavitation as an imperative technology for the treatment of petroleum refinery effluent. Journal of Water Process Engineering, 29, 100768.

21. Huang, D., Wang, Z., Zhang, J., Feng, J., Zheng, Z. and Zhang, J., 2016. Gamma radiolytic degradation of 3, 4-dichloroaniline in aqueous solution. Separation and Purification Technology, 170, 264-271.

22. Homlok, R., Takács, E. and Wojnárovits, L., 2012. Ionizing radiation induced reactions of 2, 6dichloroaniline in dilute aqueous solution. Radiation Physics and Chemistry, 81(9), 1499-1502.

23. Kahl, T., Schröder, K.W., Lawrence, F.R., Marshall, W.J., Höke, H. and Jäckh, R., 2000. Aniline. Ullmann's Encyclopedia of Industrial Chemistry.

24. Alkhuraiji, T.S. and Alkhuraiji, W.S., 2019. Detailed study of water radiolysis-based degradation of chloroorganic pollutants in aqueous solutions. Journal of hazardous materials, 368, 569-577.

25. Sarvothaman, V., 2020. Hydrodynamic Cavitation for Effluent Treatment: Using Vortex-Based Cavitation Devices, Chapter 5: Influence of scale on vortex-based cavitation. PhD. Queen's University Belfast.

26. Sarvothaman, V.P., Simpson, A.T. and Ranade, V.V., 2019. Modelling of vortex based hydrodynamic cavitation reactors. Chemical Engineering Journal, 377, 119639. 


\section{TOC Graphics}

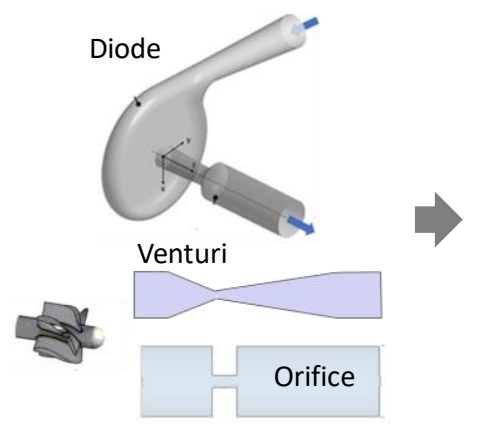

With or without swirler

Hydrodynamic Cavitation Devices based on Linear \& Swirling Flows

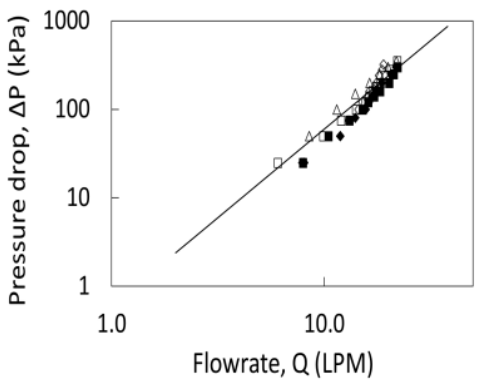

Exhibiting almost the same pressure drop versus flow rate

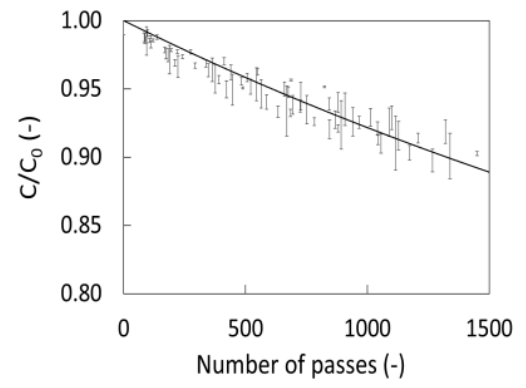

Similar performance for degradation of DCA in water 